\title{
Global Sensitivity Analysis for the Short-term Prediction of System Variables
}

\author{
Qun Zhou, Student Member, IEEE, Leigh Tesfatsion, Member, IEEE, and Chen-Ching Liu, Fellow, IEEE
}

\begin{abstract}
Short-term prediction of system variables with respect to load levels is highly important for market operations and demand response programs in wholesale power markets with congestion managed by locational marginal prices (LMPs). Previous studies have conducted local sensitivity analyses for LMPs at specific system operating points. This study undertakes a more global analysis of system variable sensitivities when LMPs are derived from DC optimal power flow solutions for day-ahead energy markets. The possible system states are first partitioned into subsets ("system patterns") based on relatively slow-changing attributes. It is next established analytically that there is a fixed linear-affine mapping between bus load patterns and corresponding system variables, conditional on a particular system pattern. It is then explained how this global piecewise linear-affine mapping can be used to predict system patterns corresponding to forecasted load patterns, hence also dispatch levels, LMPs, and line flows. A 5-bus case study is used to illustrate the accuracy of the proposed prediction method.
\end{abstract}

Index Terms-Wholesale power market, locational marginal price, global sensitivity analysis, prediction of system variables, system patterns, 5-bus illustration.

\section{INTRODUCTION}

$\mathbf{I}$ $\mathrm{N}$ many energy regions, congestion in wholesale power markets is now managed by Locational Marginal Prices $(L M P S)$, the pricing of power in accordance with the location and timing of its injection into or withdrawal from the transmission grid. The short-term prediction of LMPs in these regions is important for the daily decision-making of market participants and market operators, and many researchers have focused on this issue.

On the other hand, to provide an adequate basis for daily decision making, short-term LMP predictions need to be supplemented by predictions for other important system variables as well. These system variables include unit dispatch levels, line flows, and line congestion. Short-term predictions for these system variables assist market participants to schedule maintenance, and to trade electricity both physically and financially. They are also essential to market operators for managing congestion [1] and demand response programs [2].

Short-term LMP prediction is currently implemented primarily by means of statistical and artificial intelligence models [3]-[6]. These models are designed to capture strong correlations in daily LMPs. However, they do not consider the

Latest Revision: 27 January 2010. This study is sponsored by the Electrical Power Research Center (EPRC), Iowa State University, USA.

Qun Zhou is with the Department of Electrical and Computer Engineering, Iowa State University, Ames, IA, 50010, USA. E-mail: qzhou@iastate.edu.

Leigh Tesfatsion is with the Department of Economics, Iowa State University, Ames, IA, 50010, USA. E-mail: tesfatsi@iastate.edu

Chen-Ching Liu is with School of Electrical, Electronic and Mechanical Engineering, University College Dublin, Ireland. E-mail: liu@ucd.ie structure of wholesale power markets, hence they are difficult to use for the prediction of congestion and other system variables.

On the other hand, structural models are able to capture the characteristics of wholesale power markets and are therefore able to predict system variables [7]-[8]. However, these models require very intensive data inputs, and they are typically designed for long-term planning.

In this study we propose a method for the short-term prediction of LMPs, dispatch levels, line flows, and line congestion for day-ahead energy markets. This method exploits structural system features yet requires far less input data than typical structural modeling approaches. The method focuses on load as the main determining factor for LMPs and other system variables [3], and exploits the fact that supply-side and network topology factors are relatively stable over short periods of time.

How LMPs change with small changes in load was partly answered by the local sensitivity analysis in Conejo et al. [9]. $\mathrm{Li}$ and Bo [10] examine LMP variation in response to load variation, and they address the issue that sensitivities change when a new binding constraint occurs. However, the authors also assume that a system growth pattern exists and that load growth at each bus is proportional to this pattern. Most U.S. wholesale power markets operating under LMP are geographically large, hence distributed loads do not necessarily exhibit proportional growth.

In contrast, this study derives a global piecewise linearaffine mapping between distributed load and system outcomes for arbitrary load distributions. Consequently, sensitivity analysis is not restricted to small changes, and it is not restricted to loads changing in strict proportion. Moreover, the global sensitivity analysis does not require information about the supply offers of the generation units but instead essentially estimates these supply offers implicitly. One of the benefits of this approach is that supply offer information is typically either not publicly available [11] or released only with several months delay [12].

The remainder of this study is organized as follows. Preliminary concepts needed for understanding the proposed global sensitivity method are developed in Section II. The method itself is presented in Section III. Section IV summarizes the data requirements, procedural steps, and prediction outputs of the proposed global sensitivity prediction method. Section V illustrates the method using a 5-bus case study implemented using the AMES Wholesale Power Market Test Bed [13]. Concluding remarks are provided in Section VI. 


\section{The Sensitivity Matrix and System Patterns}

This section introduces two concepts important for the development of our global sensitivity prediction method: the sensitivity matrix, and system patterns. In Section III it will be shown that these two concepts are closely connected, in the sense that a system pattern corresponds to a unique constant sensitivity matrix.

\section{A. Sensitivity Matrix}

As is well known, LMPs are dual variables arising in optimal power flow problems, conditional on a given network topology. Specifically, they are the shadow prices corresponding to the nodal balance constraints that impose Kirchhoff's circuit law at each bus.

In the short run, when fuel prices and other supply conditions are relatively stable, load is one of the most significant factors driving the determination of LMPs. In this study we focus on the relationship between load and LMPs in day-ahead energy markets in the short run, assuming given supply offers. Our assumption that supply offers do not exhibit significant changes over suitably short time horizons is supported by an empirical examination of the supply offer data available for various ISOs in the U.S. We also take as given the network topology.

As will be shown more carefully in Section III below, under these assumptions the vector of LMPs can be expressed as a function of the vector of distributed loads:

$$
\begin{aligned}
\overline{\mathbf{L M P}} & =\mathbf{F}(\overline{\mathbf{L}}) \\
\overline{\mathbf{L M P}} & =\left[L M P_{1}, L M P_{2}, \ldots, L M P_{N}\right]^{T} \\
\overline{\mathbf{L}} & =\left[L_{1}, L_{2}, \ldots, L_{N}\right]^{T}
\end{aligned}
$$

In these equations, $N$ denotes the number of buses, $\mathrm{LMP}_{i}$ denotes the LMP at bus $i$, and $\mathrm{L}_{i}$ denotes the load at bus $i$.

The Jacobian matrix corresponding to equation (1) is

$$
\mathbf{J}=\left[\begin{array}{ccc}
\frac{\partial F_{1}}{\partial L_{1}} & \cdots & \frac{\partial F_{1}}{\partial L_{N}} \\
\vdots & \ddots & \vdots \\
\frac{\partial F_{N}}{\partial L_{1}} & \cdots & \frac{\partial F_{N}}{\partial L_{N}}
\end{array}\right]
$$

This Jacobian matrix is derived at a system operating point for a given distributed load pattern. The linear approximation of function (1) at this operating point can be expressed as follows:

$$
\Delta \overline{\mathbf{L M P}}=\mathbf{J} \cdot \Delta \overline{\mathbf{L}}
$$

Moreover, the derivation of the Jacobian matrix (4) can be extended to encompass rates of change with regard to unit dispatch levels, transmission line power flows, and line shadow prices, in addition to LMPs. In the remainder of this study, the term "sensitivity matrix" will refer to this extended Jacobian matrix.

\section{B. System Pattern}

In this study we characterize a system pattern in terms of the dispatch states of generation units and the congestion states of transmission lines. Specifically, each generation unit is categorized as dispatched at minimum capacity, dispatched at maximum capacity, or partially dispatched (implying the unit is marginal). Moreover, adopting the convention that the direction of power flow in a transmission line is positive, each line is categorized as positively congested, negatively congested, or not congested.

The flags used to denote the states of generation units and transmission lines are displayed in Table I. Note that a

TABLE I

FLAGS USED FOR SYSTEM PATTERNS

\begin{tabular}{ccccccc}
\hline & \multicolumn{3}{c}{ units } & \multicolumn{3}{c}{ transmission lines } \\
\hline State & $\begin{array}{l}\text { Minimum } \\
\text { capacity }\end{array}$ & $\begin{array}{l}\text { Marginal } \\
\text { unit }\end{array}$ & $\begin{array}{l}\text { Maximum } \\
\text { capacity }\end{array}$ & $\begin{array}{l}\text { Negative } \\
\text { congestion }\end{array}$ & $\begin{array}{c}\text { No } \\
\text { congestion }\end{array}$ & $\begin{array}{c}\text { Positive } \\
\text { congestion }\end{array}$ \\
\hline Flag & -1 & 0 & 1 & -1 & 0 & 1 \\
\hline
\end{tabular}

system pattern does not determine the exact dispatch level of marginal units or the exact power flows over non-congested lines. Consequently, multiple operating points can map into any system pattern for which at least one unit is marginal or at least one line is not congested.

\section{Global Sensitivity Method}

Making use of the concepts introduced in Section II, this section uses sensitivity analysis to establish the existence of a global piecewise-linear relationship between distributed loads and system variables. This mapping is derived under mild regularity conditions, assuming a given network topology and given supply offers for generation units.

We first present a standard DC optimal power flow $(D C O P F)$ problem as set out in [14], expressed in a form suitable for our purposes. As in [9], we then perturb the Karush-Kuhn-Tucker (KKT) first-order necessary conditions for this DCOPF problem, conditional on a given system pattern, and demonstrate the existence of a linear mapping between distributed loads and system variables for this pattern.

\section{A. DCOPF Problem Formulation}

Consider a standard DCOPF problem for a particular hour $\mathrm{H}$ of a day-ahead energy market, as formulated in [14]. Line flows are expressed in linear form making use of shift factors $S_{i j}$, where $S_{i j}$ gives the change in the power flow for line $j$ with respect to a change in the power injection at bus $i$ [15]. Lower and uppper limits for line $j$ are denoted by $F_{j}^{-}$and $F_{j}^{+}$. The number of buses is denoted by $N$ and the number of transmission lines is denoted by $T$.

For simplicity, we assume there is a single load $L_{i}$ (in MWs) and a single generation unit $G_{i}$ at each bus $i$. The cost function of $G_{i}$ for hour $\mathrm{H}$ is assumed to take the quadratic form

$$
C_{i}\left(P_{i}\right)=a_{i} P_{i}+b_{i} P_{i}^{2}(\$ / h),
$$

where $P_{i}$ denotes real power in MWs. The supply offer submitted by $G_{i}$ to the ISO for hour H consists of its marginal cost function $\mathrm{MC}_{i}\left(P_{i}\right)=a_{i}+2 b_{i} P_{i}(\$ / \mathrm{MWh})$, derived from (6), together with its hour-H minimum and maximum operating capacity limits $\operatorname{Cap}_{i}^{L}$ and $\operatorname{Cap}_{i}^{U}$ in MWs.

Given these assumptions and notations, the DCOPF problem for hour $\mathrm{H}$ can be expressed as follows. Dual variables are 
indicated after their corresponding constraints, separated by colon marks.

$$
\begin{aligned}
\min _{\mathbf{P}} \sum_{i=1}^{N}\left[a_{i} P_{i}+b_{i} P_{i}^{2}\right] & \\
\text { s.t. } \sum_{i=1}^{N} P_{i}-\sum_{i=1}^{N} L_{i} & =0: \lambda \\
\sum_{i=1}^{N} S_{i j}\left[P_{i}-L_{i}\right] & \leq F_{j}^{+}: \mu_{j}^{+}, \\
\text {for } j & =1: T \\
\sum_{i=1}^{N} S_{i j}\left[P_{i}-L_{i}\right] & \leq-F_{j}^{-}: \mu_{j}^{-}, \\
\text {for } j & =1: T \\
P_{i} \leq \operatorname{Cap}_{i}^{U}: \gamma_{i}^{+}, \quad \text { for } i & =1: N \\
-P_{i} \leq \operatorname{Cap}_{i}^{L}: \gamma_{i}^{-}, \text {for } i & =1: N
\end{aligned}
$$

As is well known, for this lossless DCOPF problem the LMP at bus $i$ can equivalently be expressed as the sum of energy and congestion components:

$$
L M P_{i}=\lambda_{i}+\sum_{j=1}^{T} S_{i j} \mu_{j}^{+}+\sum_{j=1}^{T} S_{i j} \mu_{j}^{-}
$$

Note that the coefficients for the inequality constraints in this DCOPF problem are independent of the distributed loads. Consequently, to ease subsequent analysis, it is useful to reexpress this problem in a more compact form in which the inequality constraints are divided into two types, unit capacity constraints and line limits, each expressed in vector form with constant coefficients. Letting $\overline{\mathbf{C}}$ denote the vector of unit capacity limits and $\overline{\mathbf{F}}$ denote the vector of line thermal limits, this compact form is as follows:

$$
\begin{aligned}
& \min _{\mathbf{P}} \sum_{i=1}^{N}\left[a_{i} P_{i}+b_{i} P_{i}^{2}\right] \\
& \text { s.t. } \quad \sum_{i=1}^{N} P_{i}-\sum_{i=1}^{N} L_{i}=0: \quad \lambda \\
& \bar{\alpha} \cdot \overline{\mathbf{P}} \leq \overline{\mathbf{C}}: \quad \mu \\
& \bar{\beta} \cdot \overline{\mathbf{P}}-\bar{\beta} \cdot \overline{\mathbf{L}} \leq \overline{\mathbf{F}}: \quad \sigma
\end{aligned}
$$

where

$$
\begin{aligned}
\bar{\alpha} & =\left[\begin{array}{cccc}
\alpha_{11} & \alpha_{21} & \cdots & \alpha_{N 1} \\
\alpha_{12} & \alpha_{22} & \cdots & \alpha_{N 2} \\
\vdots & \vdots & \alpha_{i k} & \vdots \\
\alpha_{1(2 N)} & \alpha_{2(2 N)} & \cdots & \alpha_{N(2 N)}
\end{array}\right] \\
\bar{\beta} & =\left[\begin{array}{cccc}
\beta_{11} & \beta_{21} & \cdots & \beta_{N 1} \\
\beta_{12} & \beta_{22} & \cdots & \beta_{N 2} \\
\vdots & \vdots & \beta_{i k} & \vdots \\
\beta_{1(2 T)} & \beta_{2(2 T)} & \cdots & \beta_{N(2 T)}
\end{array}\right] \\
\overline{\mathbf{P}} & =\left[P_{1}, P_{2}, \ldots, P_{N}\right]^{T} \\
\overline{\mathbf{L}} & =\left[L_{1}, L_{2}, \ldots, L_{N}\right]^{T} \\
\overline{\mathbf{C}} & =\left[C_{1}, C_{2}, \ldots, C_{2 N}\right]^{T} \\
\overline{\mathbf{F}} & =\left[F_{1}, F_{2}, \ldots, F_{2 T}\right]^{T}
\end{aligned}
$$

\section{B. Perturbation Analysis of KKT Conditions}

The Lagrangian function for the compact DCOPF problem formulation (14) can be expressed as

$$
\begin{aligned}
\mathcal{L}= & \sum_{i=1}^{N}\left[a_{i} P_{i}+b_{i} P_{i}^{2}\right]+\lambda \sum_{i=1}^{N}\left[P_{i}-L_{i}\right] \\
& +\sum_{k=1}^{2 N} \mu_{k}\left(\sum_{i=1}^{N} \alpha_{i k} P_{i}-C_{k}\right) \\
& +\sum_{k=1}^{2 T} \sigma_{k}\left(\sum_{i=1}^{N} \beta_{i k}\left[P_{i}-L_{i}\right]-F_{k}\right)
\end{aligned}
$$

The first-order necessary KKT conditions for this problem are as follows:

$$
\begin{aligned}
a_{i}+2 b_{i} P_{i}+\lambda+\sum_{k=1}^{2 N} \mu_{i} \alpha_{i k} & \\
+\sum_{k=1}^{2 T} \sigma_{k} \beta_{i k} & =0, \quad i=1: N \\
\sum_{i=1}^{N}\left[P_{i}-L_{i}\right] & =0, \\
\mu_{k} \sum_{i=1}^{N}\left[\alpha_{i k} P_{i}-C_{k}\right] & =0, \\
\sum_{i=1}^{N}\left[\alpha_{i k} P_{i}-C_{k}\right] & \leq 0, \\
\mu_{k} & \geq 0, \quad k=1: 2 N \\
\sigma_{k} \sum_{i=1}^{N}\left(\beta_{i k}\left[P_{i}-L_{i}\right]-F_{k}\right) & =0, \\
\sum_{i=1}^{N}\left(\beta_{i k}\left[P_{i}-L_{i}\right]-F_{k}\right) & \leq 0, \\
\sigma_{k} & \geq 0, \quad k=1: 2 T
\end{aligned}
$$

For each category of constraints, let the number of binding and non-binding constraints be denoted as indicated in Table II.

TABLE II

NUMBER OF BINDING AND NON-BINDING CONSTRAINTS

\begin{tabular}{clll}
\hline Constraint & Binding & Non-Binding & Slack \\
Category & Constraints & Constraints & Variables \\
\hline Unit Cap & First $H$ & $2 N-H$ & $S_{k}$ \\
Line Limit & First $M$ & $2 T-M$ & $V_{k}$ \\
\hline
\end{tabular}

The shadow price for a non-binding constraint is zero. For such constraints, slack variables are introduced for sensitivity analysis. Using the particular slack-variable notation introduced in Table II, the KKT conditions can be expressed in the following equivalent form:

$$
\begin{aligned}
a_{i}+2 b_{i} P_{i}+\lambda & +\sum_{k=1}^{H} \mu_{k} \alpha_{i k} \\
& +\sum_{k=1}^{M} \sigma_{k} \beta_{i k}=0, \quad i=1: N
\end{aligned}
$$




$$
\begin{aligned}
\sum_{i=1}^{N} P_{i}-L_{i} & =0 \\
\sum_{i=1}^{N} \alpha_{i k} P_{i}-C_{k} & =0 \\
\mu_{k} \geq 0, \quad k & =1: H \\
\sum_{i=1}^{N} \alpha_{i k} P_{i}-C_{k} & =-S_{k}, \\
\mu_{k}=0, \quad k & =H+1: 2 N \\
\sum_{i=1}^{N} \beta_{i k} P_{i}-\sum_{i=1}^{N} \beta_{i k} L_{i}-F_{k} & =0, \\
\sigma_{k} \geq 0, \quad k & =1: M \\
\sum_{i=1}^{N} \beta_{i k} P_{i}-\sum_{j=1}^{N} \beta_{i k} L_{i}-F_{k} & =-V_{k}, \\
\sigma_{k}=0, \quad k & =M+1: 2 T
\end{aligned}
$$

Totally differentiating the KKT conditions (16)-(21) gives the following sensitivity relationships:

$$
\begin{aligned}
2 b_{i} d P_{i}+d \lambda & +\sum_{k=1}^{H} \alpha_{i k} d \mu_{k} \\
+\sum_{k=1}^{M} \beta_{i k} d \sigma_{k} & =0, \quad i=1: N \\
\sum_{i=1}^{N}\left(d P_{i}-d L_{i}\right) & =0
\end{aligned}
$$

$$
\begin{aligned}
\sum_{i=1}^{N} \alpha_{i k} d P_{i} & =0, \quad k=1: H \\
\sum_{i=1}^{N} \beta_{i k} d P_{i} & =\sum_{i=1}^{N} \beta_{i k} d L_{i}, \\
k & =1: M \\
\sum_{i=1}^{N} d P_{i} & =-d S_{k}, \\
k & =H+1: 2 N \\
\sum_{i=1}^{N} \beta_{i k} d P_{i} & =\sum_{i=1}^{N} \beta_{i k} d L_{i}-d V_{k}, \\
k & =M+1: 2 T
\end{aligned}
$$

Suppose the load $L_{j}$ at bus $j$ is perturbed while the loads at all other buses remain unchanged, i.e., $\mathrm{d} L_{j} \neq 0$ and $\mathrm{d} L_{i}=0$ for $i \neq j$. Dividing the sensitivity relationships by $\mathrm{d} L_{j}$, the results are expressed in matrix form in (28) and (29). In these two matrix relationships, note that the coefficient matrices multiplying the sensitivity vectors are independent of the distributed load pattern.

\section{Linear Relationship Verification}

In Zhou et al. [16] it is demonstrated that a sufficient regularity condition guaranteeing the invertibility of the coefficient matrix in (28) is that ${ }^{1}$

$$
H+M+1 \leq N
$$

${ }^{1}$ Although similar matrices are inverted in [9] and [10], conditions for invertibility are not considered.

$$
\begin{aligned}
& {\left[\begin{array}{cccc|ccccccccc}
2 b_{1} & 0 & \cdots & 0 & 1 & \alpha_{11} & \alpha_{12} & \cdots & \alpha_{1 H} & \beta_{11} & \beta_{12} & \cdots & \beta_{1 M} \\
0 & 2 b_{2} & \cdots & 0 & 1 & \alpha_{21} & \alpha_{22} & \cdots & \alpha_{2 H} & \beta_{21} & \beta_{22} & \cdots & \beta_{2 M} \\
\vdots & \vdots & \ddots & \vdots & \vdots & \vdots & \vdots & \ddots & \vdots & \vdots & \vdots & \ddots & \vdots \\
0 & 0 & \cdots & 2 b_{N} & 1 & \alpha_{N 1} & \alpha_{N 2} & \cdots & \alpha_{N H} & \beta_{N 1} & \beta_{N 2} & \cdots & \beta_{N M} \\
\hline 1 & 1 & \cdots & 1 & 0 & 0 & 0 & \cdots & 0 & 0 & 0 & \cdots & 0 \\
\alpha_{11} & \alpha_{21} & \cdots & \alpha_{N 1} & 0 & 0 & 0 & \cdots & 0 & 0 & 0 & \cdots & 0 \\
\alpha_{12} & \alpha_{22} & \cdots & \alpha_{N 2} & 0 & 0 & 0 & \cdots & 0 & 0 & 0 & \cdots & 0 \\
\vdots & \vdots & \ddots & \vdots & \vdots & \vdots & \vdots & \ddots & \vdots & \vdots & \vdots & \ddots & \vdots \\
\alpha_{1 H} & \alpha_{2 H} & \cdots & \alpha_{N H} & 0 & 0 & 0 & \cdots & 0 & 0 & 0 & \cdots & 0 \\
\beta_{11} & \beta_{21} & \cdots & \beta_{N 1} & 0 & 0 & 0 & \cdots & 0 & 0 & 0 & \cdots & 0 \\
\beta_{12} & \beta_{22} & \cdots & \beta_{N 2} & 0 & 0 & 0 & \cdots & 0 & 0 & 0 & \cdots & 0 \\
\vdots & \vdots & \ddots & \vdots & \vdots & \vdots & \vdots & \ddots & \vdots & \vdots & \vdots & \ddots & \vdots \\
\beta_{1 M} & \beta_{2 M} & \cdots & \beta_{N M} & 0 & 0 & 0 & \cdots & 0 & 0 & 0 & \cdots & 0
\end{array}\right] \cdot\left[\begin{array}{c}
d P_{1} / d L_{j} \\
d P_{2} / d L_{j} \\
\vdots \\
d P_{N} / d L_{j} \\
\hline d \lambda / d L_{j} \\
d \mu_{1} / d L_{j} \\
d \mu_{2} / d L_{j} \\
\vdots \\
d \mu_{H} / d L_{j} \\
d \sigma_{1} / d L_{j} \\
d \sigma_{2} / d L_{j} \\
\vdots \\
d \sigma_{M} / d L_{j}
\end{array}\right]=\left[\begin{array}{c}
0 \\
0 \\
\vdots \\
0 \\
0 \\
0 \\
0 \\
\beta_{j 1} \\
\beta_{j 2} \\
\vdots \\
\beta_{j M}
\end{array}\right]} \\
& {\left[\begin{array}{cccc}
\alpha_{1(H+1)} & \alpha_{2(H+1)} & \cdots & \alpha_{N(H+1)} \\
\alpha_{1(H+2)} & \alpha_{2(H+2)} & \cdots & \alpha_{N(H+2)} \\
\vdots & \vdots & \ddots & \vdots \\
\alpha_{1(2 N)} & \alpha_{2(2 N)} & \cdots & \alpha_{N(2 N)} \\
\beta_{1(M+1)} & \beta_{2(M+1)} & \cdots & \beta_{N(M+1)} \\
\beta_{1(M+2)} & \beta_{2(M+2)} & \cdots & \beta_{N(M+2)} \\
\vdots & \vdots & \ddots & \vdots \\
\beta_{1(2 T)} & \beta_{2(2 T)} & \cdots & \beta_{N(2 T)}
\end{array}\right] \cdot\left[\begin{array}{c}
d P_{1} / d L_{j} \\
d P_{2} / d L_{j} \\
\vdots \\
d P_{N} / d L_{j}
\end{array}\right]=\left[\begin{array}{c}
-d S_{H+1} / d L_{j} \\
-d S_{H+2} / d L_{j} \\
\vdots \\
-d S_{2 N} / d L_{j} \\
\beta_{j(M+1)}-d V_{M+1} / d L_{j} \\
\beta_{j(M+2)}-d V_{M+2} / d L_{j} \\
\vdots \\
\beta_{j(2 T)}-d V_{2 T} / d L_{j}
\end{array}\right]}
\end{aligned}
$$


The regularity condition (30) is normally satisfied in power system operation and is hereafter assumed to hold.

Given the invertibility of the coefficient matrix in (28), the solutions for the indicated system variable variations can be determined as linear functions of the load variations, conditional on the system pattern $s$. As indicated in (31) and (32), these solutions can be expressed as components $J_{i j}^{v s}$ of a column vector, where: $s$ denotes the underlying system pattern; $v$ indicates the relevant subvector of system variables, either the unit dispatch levels $P$, the transmission line flows $F$, the shadow prices $\lambda$ or $\mu$, or the LMPs; $i$ denotes the $i$ th element in the specified system variable subvector; and $j$ is the index for the perturbed load. Relations (31) and (32) illustrate the particular components corresponding to unit dispatch levels $P$ and transmission line flows $F$.

$$
\begin{aligned}
{\left[\begin{array}{c}
d P_{1} / d L_{j} \\
d P_{2} / d L_{j} \\
\vdots \\
d P_{N} / d L_{j}
\end{array}\right] } & =\left[\begin{array}{c}
J_{1 j}^{P s} \\
J_{2 j}^{P s} \\
\vdots \\
J_{N j}^{P s}
\end{array}\right] \\
{\left[\begin{array}{c}
d V_{M+1} / d L_{j} \\
d V_{M+2} / d L_{j} \\
\vdots \\
d V_{2 T} / d L_{j}
\end{array}\right] } & =\left[\begin{array}{c}
J_{M+1, j}^{F s} \\
J_{M+2, j}^{F s} \\
\vdots \\
J_{2 T, j}^{F s}
\end{array}\right]
\end{aligned}
$$

Suppose each value in the load interval from $L_{j}^{0}$ to $L_{j}$ gives rise to the same system pattern $s$. Let $P_{i}$ and $P_{i}^{0}$ denote the unit dispatch solutions for bus $i$ for the given loads $L_{j}^{0}$ and $L_{j}$, respectively. Integrating both sides of equations (31) and (32) with respect to $\mathrm{d} L_{j}$, one obtains:

$$
\begin{aligned}
\int_{L_{j}^{0}}^{L_{j}} \frac{d P_{i}}{d L_{j}} \cdot d L_{j} & =\int_{L_{j}^{0}}^{L_{j}} J_{i j}^{P s} \cdot d L_{j} \\
P_{i}-P_{i}^{0} & =J_{i j}^{P s}\left[L_{j}-L_{j}^{0}\right] \\
P_{i} & =J_{i j}^{P s} L_{j}+\left[P_{i}^{0}-J_{i j}^{P s} L_{j}^{0}\right]
\end{aligned}
$$

It then follows by superposition that the vector $\overline{\mathbf{P}}$ of unit dispatch solutions $P_{i}$ corresponding to a system pattern $s$ can be expressed as a linear-affine function of the distributed load vector $\overline{\mathbf{L}}$, as follows:

$$
\overline{\mathbf{P}}=\mathbf{J}^{\mathrm{Ps}} \cdot \overline{\mathbf{L}}+\overline{\mathbf{O}}^{\mathrm{Ps}},
$$

where the $i j$ th element of the $N \times N$ matrix $\mathbf{J}^{\mathbf{P s}}$ is given by $J_{i j}^{P s}$ and the $i$ th element of the $N \times 1$ vector $\overline{\mathbf{O}}^{P s}$ is given by

$$
O_{i}^{P s}=P_{i}^{s}-\sum_{j=1}^{N}\left[J_{i j}^{P s} L_{j}^{s}\right]
$$

In (37), $\overline{\mathbf{L}} \mathbf{s}=\left[L_{1}^{s}, \ldots, L_{N}^{s}\right]$ denotes any given load pattern associated with the system pattern $s$, and $\overline{\mathbf{P}^{\mathbf{s}}}=\left[P_{1}^{s}, \ldots, P_{N}^{s}\right]$ denotes the dispatch vector corresponding to this given load pattern. For example, as will be seen below, $\left(\overline{\mathbf{L}}^{\mathbf{s}}, \overline{\mathbf{P}}^{\mathbf{s}}\right)$ could represent an historical load-dispatch outcome that was observed to be associated with system pattern $s$.

Clearly the same sequence of steps can also be used to derive an $s$-conditional linear-affine relationship for each other type of system variable as well. The full matrix $\mathbf{J}^{\mathbf{s}}$ consisting of block matrices $\mathbf{J}^{\mathbf{v s}}$, one block for each system variable type $v$, will be referred to as the sensitivity matrix corresponding to system pattern $s$. Similarly, the full vector $\overline{\mathbf{O}}^{\mathbf{s}}$ consisting of subvectors $\overline{\mathbf{O}}^{\mathbf{v s}}$, one subvector for each system variable type $v$, will be referred to as the ordinate vector corresponding to the system pattern $s$.

In summary, given the regularity condition (30), all of the above derivations are reversible. It follows that there is a one-to-one mapping between the system pattern $s$ and the sensitivity matrix $\mathbf{J}^{\mathbf{s}}$. Moreover, for each system pattern $s$, the vector of system variables is a linear-affine function of the distributed load vector with coefficient matrix given by $\mathbf{J}^{\mathbf{s}}$. Consequently, from a global system perspective, the system variables are piecewise linear-affine functions of distributed load with break points determined by switch points in the system pattern $s$.

\section{Prediction Method: General Approach}

The finding in Section III of a constant sensitivity matrix $\mathbf{J}^{s}$ for a given system pattern $s$ provides a theoretical foundation for the prediction of system variables in day-ahead energy markets managed by LMP. The proposed prediction method requires data input sufficient to permit the recognition of historical system patterns $s$ and the estimation of a sensitivity matrix $\mathbf{J}^{s}$ and ordinate vector $\overline{\mathbf{O}}^{s}$ for each of these historical patterns $s$. These historical data requirements are as follows:

\section{Historical Data Requirements:}

- Ex-post dispatch levels for each generation unit

- Ex-post power flows for each transmission line

- Capacity limits for each generation unit

- Ex-post and forecasted loads at each bus

- Ex-post LMPs at each bus

Given this historical data, the following aspects can be predicted over the short run.

\section{Short-Run Predictions:}

- Dispatch level for each unit

- Power flow for each line

- LMP at each bus

- Congested lines

More precisely, we propose the prediction method outlined via a flow diagram in Fig. 1. This prediction method consists of four principal steps:

1) Step 1 -Determine historical system patterns: This step involves the collection of the required historical data, and the use of this data to identify historical system patterns $s$.

2) Step 2 - Estimate sensitivity matrices and ordinate vectors: This step involves the estimation of a sensitivity matrix $\mathbf{J}^{\mathbf{s}}$ and ordinate vector $\overline{\mathbf{O}}^{\mathbf{s}}$ for each historical system pattern $s$, making use of actual operating points to determine the ordinate vectors.

3) Step 3 - Determine current system pattern: This step involves the iterative determination of the system pattern for the current distributed load pattern, meaning that predicted system variables must be consistent ex post with the system pattern that is ex ante assumed. 
4) Step 4 - Predict system variables: After the current system pattern $s$ is found, the system variables can be predicted by means of the corresponding sensitivity matrix $\left(\mathbf{J}^{\mathbf{s}}\right.$ and ordinate vector $\overline{\mathbf{O}}^{\mathbf{s}}$.

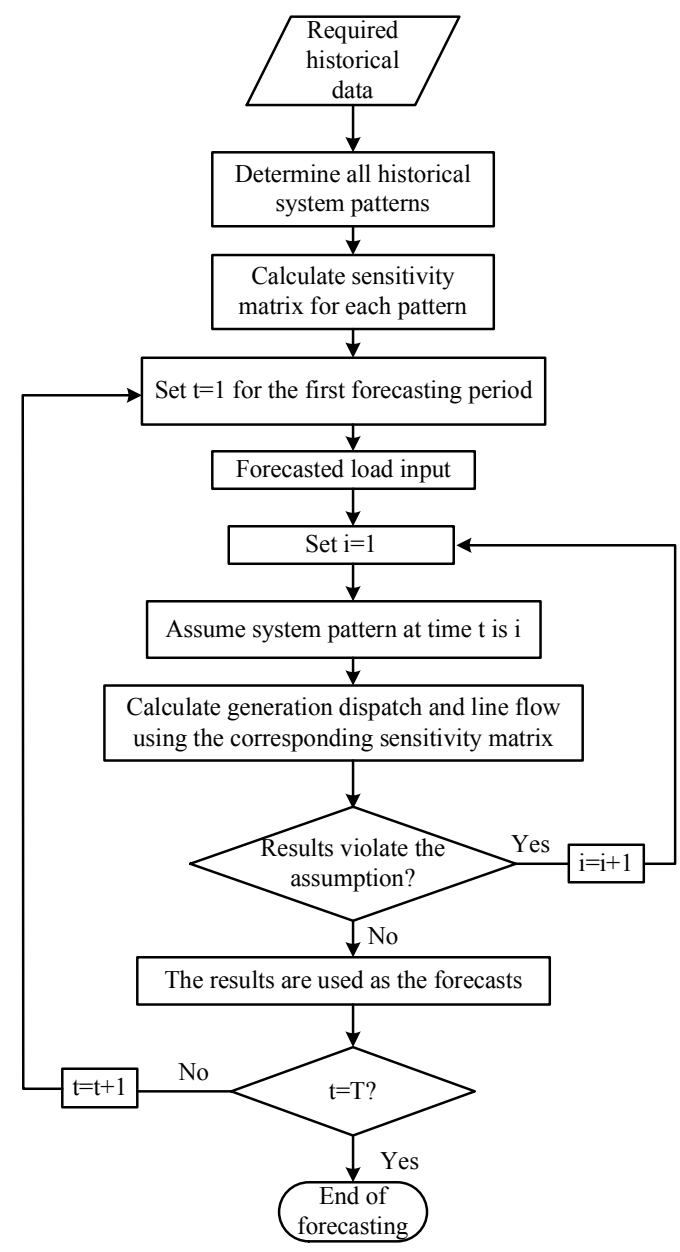

Fig. 1. Flow diagram for proposed prediction method

\section{Prediction Illustration for a 5-Bus System}

The input data file for the 5-bus test case included in the download of the AMES Wholesale Power Market Test Bed [17] is used below to illustrate the prediction method outline in Section IV. As depicted in Fig. 2, this 5-bus test case has six transmission lines (TL1-TL6), five generation units (G1-G5), and three load-serving entities (LSE 1-LSE 3).

The AMES test bed implements a wholesale power market operating over a transmission network with congestion managed by LMP [13]. Profit-seeking generation units in AMES are able to learn over time how to report their supply offers based on their past profit outcomes. In this study, however, we assume that each generation unit reports its true cost and capacity attributes to the ISO each day for the day-ahead energy market.

As detailed in [16], the load data for our 5-bus case study are scaled-down time-varying loads derived from load data available at the MISO website [11]. Using this load data, we ran AMES for 365 simulated days in order to determine the

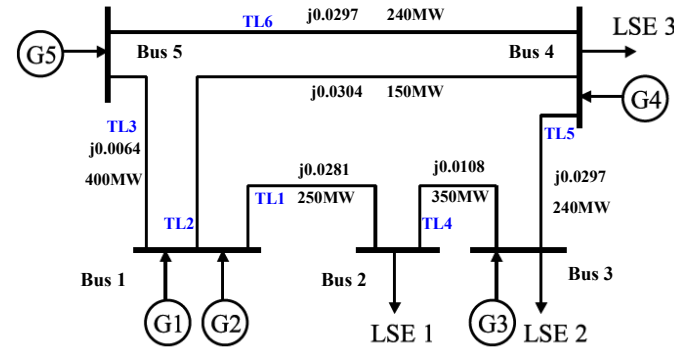

Fig. 2. 5-bus network

historical system patterns $s$ (Step 1). The sensitivity matrix and ordinate vector for each of these patterns was then calculated (Step 2). We then carried out system pattern determination and system variable prediction for various possible distributed load patterns (Steps 3 and 4).

These steps are explained more carefully in the following subsections.

\section{A. Prediction Steps 1 and 2 for the 5-Bus Case Study}

Nine system patterns were identified from the AMES output obtained from the 365 simulated days using a year of scaleddown MISO load data. The four most frequently observed system patterns are displayed in Table III.

TABLE III

THE FOUR MOST FREQUENT HISTORICAL SYSTEM PATTERNS OBSERVED FOR THE 5-BUS CASE STUDY

\begin{tabular}{cccccccccccc}
\hline Pattern & G1 & G2 & G3 & G4 & G5 & TL1 & TL2 & TL3 & TL4 & TL5 & TL6 \\
\hline S1 & 1 & 0 & -1 & -1 & 0 & 0 & 0 & 0 & 0 & 0 & 0 \\
S2 & 0 & 0 & 0 & -1 & 0 & 1 & 0 & 0 & 0 & 0 & 0 \\
S3 & 1 & 0 & 0 & -1 & 0 & 1 & 0 & 0 & 0 & 0 & 0 \\
S4 & 1 & 0 & -1 & -1 & 0 & 1 & 0 & 0 & 0 & 0 & 0 \\
\hline
\end{tabular}

The sensitivity matrix and ordinate vector for each of the nine historical system patterns were then estimated making use of actual system operating points observed for each historical system pattern. To illustrate, we compute the sensitivity matrix and ordinate vector for the dispatch level of generation unit G1 in system pattern $\mathrm{S} 4$, meaning $i=1, v=P$, and $s=4$.

Specifically, using four historically observed operating points $t=1, \ldots, 4$ associated with system pattern $\mathrm{S} 4$, a set of four linear equations was determined as follows:

$$
\begin{aligned}
& P_{1}^{1}=J_{11}^{P 4} L_{1}^{1}+J_{12}^{P 4} L_{2}^{1}+J_{13}^{P 4} L_{3}^{1}+O_{1}^{P 4} \\
& P_{1}^{2}=J_{11}^{P 4} L_{1}^{2}+J_{12}^{P 4} L_{2}^{2}+J_{13}^{P 4} L_{3}^{2}+O_{1}^{P 4} \\
& P_{1}^{3}=J_{11}^{P 4} L_{1}^{3}+J_{12}^{P 4} L_{2}^{3}+J_{13}^{P 4} L_{3}^{3}+O_{1}^{P 4} \\
& P_{1}^{4}=J_{11}^{P 4} L_{1}^{4}+J_{12}^{P 4} L_{2}^{4}+J_{13}^{P 4} L_{3}^{4}+O_{1}^{P 4}
\end{aligned}
$$

Here $P_{1}^{t}$ denotes the dispatch level of G1 at operating point $t$ and $L_{1}^{t}$ denotes the load level of LSE $j$ at operating point $t$. These four equations determine solution values for the four unknown variables $J_{11}^{P 4}, J_{12}^{P 4}, J_{13}^{P 4}$ and $O_{1}^{P 4}$. The first three solution values determine one row of the block matrix $\mathbf{J}^{\mathbf{P 4}}$, hence also one row of the sensitivity matrix $\mathbf{J}^{\mathbf{s}}$. The last 
solution value determines one element of $\overline{\mathbf{O}}^{\mathbf{P} 4}$, hence one element of the ordinate vector $\overline{\mathrm{O}}^{4}$. Other rows and elements can be similarly computed. The sensitivity matrix and ordinate vector for S4 are partially shown in Table IV.

TABLE IV

SENSITIVITY MATRIX AND ORDINATE VECTOR FOR SYSTEM PATTERN S4 (PARTIALLY SHOWN)

\begin{tabular}{c}
{$\left[\begin{array}{c}O^{L M P} \\
23.83 \\
-3400.00 \\
-2729.20 \\
-994.43 \\
-52.79\end{array}\right]$} \\
\hline$O^{P}$ \\
{$\left[\begin{array}{c}110 \\
6679.66 \\
0 \\
0 \\
-6679.30\end{array}\right] \quad\left[\begin{array}{ccc}-0.02 & -0.03 & 0.01 \\
8.74 & 7.12 & 2.35 \\
7.02 & 5.72 & 1.89 \\
2.59 & 2.09 & 0.71 \\
-0.17 & 0.15 & 0.05\end{array}\right]$} \\
\hline $\left.\begin{array}{c}O^{F} \\
250.00 \\
601.57 \\
5938.09 \\
250.00 \\
208.97 \\
741.26\end{array}\right] \quad\left[\begin{array}{ccc}0 & J^{P} \\
-17.21 & -13.89 & -4.23 \\
0 & 0 & 0 \\
0 & 0 & 0 \\
17.92 & 14.68 & 5.14\end{array}\right]$ \\
\hline $\left.\begin{array}{ccc}0 & J^{F} \\
-1.43 & -1.05 & -0.03 \\
-15.78 & -12.84 & -4.20 \\
-1.0 & 0 & 0 \\
-0.89 & -0.92 & 0.03 \\
-2.14 & -1.84 & -0.94\end{array}\right]$
\end{tabular}

\section{B. Prediction Step 3 for the 5-Bus Case Study}

Now suppose that a certain distributed load pattern is forecasted for the near future. For example, suppose the forecasted loads for buses 1 through 3 in a particular hour $\mathrm{H}$ are $L_{1}$ $=245.50 \mathrm{MW}, L_{2}=211.64 \mathrm{MW}$, and $L_{3}=170.17 \mathrm{MW}$. An iterative assume-check procedure can then be undertaken to determine which system pattern corresponds to these forecasted load conditions.

Specifically, start by assuming a system pattern. Given this assumed system pattern, use the corresponding sensitivity matrix and ordinate vector to predict the system variables. If these predictions are consistent with the assumed system pattern, then the correct system pattern has been found. Otherwise, switch to a new assumed system pattern and again check the system variable predictions for consistency with the assumed pattern.

Since only finitely many system patterns are possible, this assume-check iterative procedure ultimately determines the correct system pattern. Table V illustrates the outcomes of this procedure for the four system patterns described in Table III.

From Table $\mathrm{V}$ it is seen that the new operating point is in system pattern S3 since the predicted system variables resulting from this assumed system pattern are consistent with the pattern. For each of the other three system patterns, the resulting predicted system variables imply at least one violation of the unit capacity and transmission line conditions implied by this pattern.
TABLE V

System VARiable Predictions For System Patterns S1-S4

\begin{tabular}{lcccc}
\hline Pattern & & & & \\
Assumption & $\mathrm{S} 1$ & $\mathrm{~S} 2$ & $\mathrm{~S} 3$ & $\mathrm{~S} 4$ \\
\hline G1 & 110.00 & 111.47 & 110.00 & 110.00 \\
G2 & 87.14 & 9.54 & 10.32 & -1204.90 \\
G3 & 0.00 & 88.36 & 88.35 & 0.00 \\
G4 & 0.00 & 0.00 & 0.00 & 0.00 \\
G5 & 430.10 & 418.03 & 418.66 & 1701.60 \\
TL1 & 297.63 & 250.00 & 250.00 & 250.00 \\
TL2 & 135.39 & 116.64 & 116.57 & 23.20 \\
TL3 & -238.59 & -245.68 & -246.24 & -1368.10 \\
TL4 & 52.13 & 4.50 & 4.50 & 4.50 \\
TL5 & -159.70 & -118.77 & -118.84 & -199.10 \\
TL6 & -191.51 & -172.32 & -172.40 & -333.50 \\
\hline Violation & Yes & Yes & No & Yes \\
\hline
\end{tabular}

\section{Prediction Step 4 for the 5-Bus Case Study}

Once the correct system pattern is found for a forecasted load pattern, the sensitivity matrix and ordinate vector for this pattern can be used to predict system variables. These predictions should be very precise, since the input data is exact. Any deviation of predictions from actual values will be due purely to computational round-off and truncation errors.

For example, consider again the forecasted loads $L_{1}=$ 245.50MW, $L_{2}=211.64 \mathrm{MW}$, and $L_{3}=170.17 \mathrm{MW}$ considered in Section V-B for a particular hour $\mathrm{H}$, for which the correct system pattern was determined to be S3. LMP and congestion predictions generated for these forecasted loads under system pattern S3 are reported in Table VI, along with the actual LMPs and congestion resulting under this load condition.

TABLE VI

LMP AND Line CONGESTION PREDICTIONS UNDER S3

\begin{tabular}{cccccc}
\hline LMPs & LMP1 & LMP2 & LMP3 & LMP4 & LMP5 \\
\hline Predicted & 15.14 & 29.50 & 26.79 & 19.29 & 15.84 \\
Actual & 15.12 & 29.49 & 26.77 & 19.28 & 15.86 \\
\hline \multicolumn{2}{l}{ Congested Lines } & \multicolumn{2}{c}{ Predicted TL1 } & \multicolumn{3}{c}{ Actual TL1 } \\
\hline
\end{tabular}

The proposed approach is also tested for the prediction of LMPs and line flows over successive hours. Figs. 3 and 4 display the predicted and actual values for the power flow on line TL1 and the LMP at bus 2 for all 24 hours of the simulated day 363. As seen, the predicted values are nearly coincident with the actual values, differing only by small computational round-off and truncation errors.

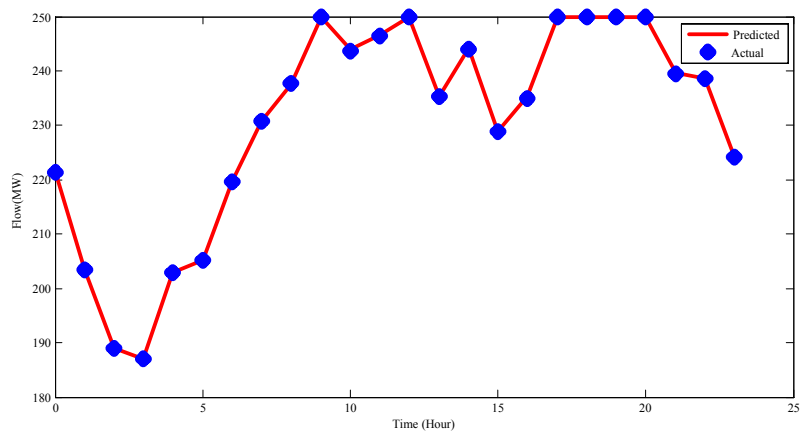

Fig. 3. Predicted Hourly Power Flow on Line TL1 During Day 363 


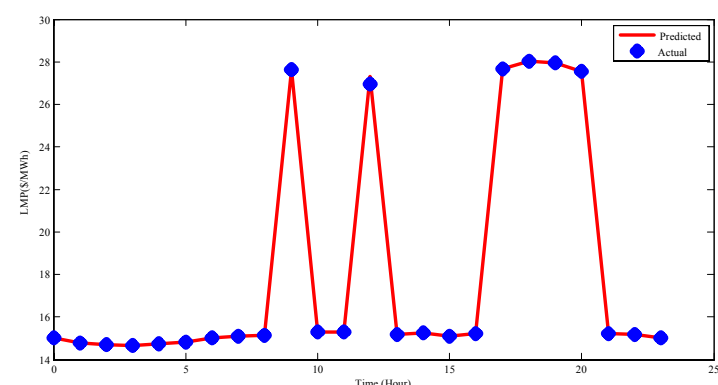

Fig. 4. Predicted LMP at Bus 2 During Day 363

\section{CONCLUSION}

Short-run system variable prediction is critical for both market participants and market operators. In this study we explore the relationship between system variables and distributed load, assuming that supply offers and network topology remain relatively static. Compared to previous research, which focuses on local conditions, we provide a global sensitivity analysis at the level of distributed loads by introducing the concept of a system pattern. We derive a piecewise linear-affine mapping between distributed loads and system variables, and we apply this mapping to predict prices and congestion conditions. A 5-bus case study is used to illustrate the effectiveness of this proposed prediction method.

Moreover, this same approach can be used by system operators in real-time operation to redispatch units at new load levels. Operation times should be reduced because our approach involves the solution of relatively straightforward systems of linear equations rather than OPF problems. Demand response programs might also benefit from the approach because it permits the rapid determination of load curtailment effects. In addition, the system pattern concept could be applied to system variable prediction in a smart grid environment where price-sensitive demand is supported by smart metering.

It is noteworthy that some prediction errors arise in the 5bus case study due to computational round-off and truncation errors. If our approach is used in large-scale systems with incomplete structural information, these prediction errors will be magnified. It might therefore be desirable to modify our method to improve accuracy for such applications. Future work will consider this issue.

\section{REFERENCES}

[1] A. Kumar, S. C. Srivastava, and S. N. Singh, "Congestion management in competitive power market: A bibliographical survey," Electrical Power Systems Research, vol. 76, pp. 153-164, 2005.

[2] P. Jazayeri, A. Schellenberg, W. D. Rosehart, J. Doudna, S. Widergren, D. Lawrence, J. Mickey, and S. Jones, "A survey of load control programs for price and system stability," IEEE Trans. Power Syst., vol. 20, no. 3, pp. 1504-1509, Aug. 2005.

[3] G. Li, C. C. Liu, C. Mattson, and J. Lawarrée, "Day-ahead electricity price forecasting in a grid environment," IEEE Trans. Power Syst., vol. 22, no. 1, pp. 266-274, Feb. 2007.

[4] F. Nogales, J. Contreras, A. Conejo, and R. Espinola,"Forecasting nextday electricity prices by time series models," IEEE Trans. Power Syst., vol. 17, no. 2, pp. 342-348, May 2002.

[5] J. Contreras, R. Espinola, F. J. Nogales, and A. J. Conejo,"ARIMA models to predict next-day electricity prices," IEEE Trans. Power Syst., vol. 18, no. 3, pp. 1014-1020, Aug. 2003.
[6] R. C. Garcia, J. Contreras, M. Akkeren, and J. B. C. Garcia,"A GARCH forecasting model to predict day-ahead electricity prices," IEEE Trans. Power Syst., vol. 20, no. 2, pp. 867-874, May 2005.

[7] D. W. Bunn, "Forecasting loads and prices in competitive power markets," Proceedings of IEEE, vol. 88, no. 2, pp. 163-169, Feb. 2000.

[8] J. Bastian, J. Zhu, V. Banunarayanan, and R. Mukerji, "Forecasting energy prices in a competitive market," IEEE computer applications in power, pp. 40-45, Jul. 1999.

[9] A. J. Conejo, E. Castillo, R. Mínguez, and F. Milano, "Locational marginal price sensitivities," IEEE Trans. Power Syst., vol. 24, no. 4, pp. 2026-2033, Nov. 2005.

[10] F. Li, and R. Bo, "Congestion and price prediction under load variation," IEEE Trans. Power Syst., vol. 24, no. 2, May 2009.

[11] MISO Website. [Online]. Available: http://www.midwestiso.org

[12] NYISO Website. [Online]. Available: http://www.nyiso.com

[13] H. Li and L. Tesfatsion, "The AMES Wholesale Power Market Test Bed: A Computational Laboratory for Research, Teaching, and Training," IEEE Proceedings, Power and Energy Society General Meeting, Calgary, Alberta, CA, July 26-30, 2009.

[14] A. J. Wood and B. F. Wollenberg, Power Generation Operation and Control, New York: Wiley, 1996.

[15] E. Litvinov. Power system and LMP fundamentals. [Online]. Available: http://www.iso-ne.com/support/training/courses/wem301/lmp.pdf

[16] Q. Zhou, L. Tesfatsion, and C. C. Liu, "Global sensitivity analysis for price and congestion forecasting," working paper, in progress.

[17] AMES Wholesale Power Market Test Bed: Homepage. [Online]. Available at http://www.econ.iastate.edu/tesfatsi/AMESMarketHome.htm

Qun Zhou received her B.E. degree from Huazhong University of Science and Technology, Wuhan, China in 2007. She is currently pursuing a Ph.D. degree at Iowa State University. Her research interests include short-term price forecasting and power system planning.

Leigh Tesfatsion received her Ph.D. degree from the University of Minnesota in 1975. She is Professor of Economics, Mathematics, and Electrical and Computer Engineering at Iowa State University. Her principal research area is agent-based test bed development, with a particular focus on restructured electricity markets. She is an active participant in IEEE PES working groups and task forces focusing on power economics issues and a co-organizer of the ISU Electric Energy Economics (E3) Group. She serves as associate editor for a number of journals, including J. of Energy Markets.

Chen-Ching Liu received his Ph.D. degree from the University of California, Berkeley. He is currently a Professor in the School of Electrical, Electronic and Mechanical Engineering at National University of Ireland, Dublin, where he also serves as Deputy Principal of the College of Engineering, Mathematical and Physical Sciences. From 2005 to 2008 he was Palmer Chair Professor of Electrical and Computer Engineering at Iowa State University, Ames. During 1983 to 2005 he was a Professor of Electrical Engineering at the University of Washington, Seattle, where he also served as an Associate Dean of Engineering from 2000 to 2005.

Dr. Liu received an IEEE Third Millennium Medal in 2000 and the IEEE Power Engineering Society Outstanding Power Engineering Educator Award in 2004. He is a Fellow of the IEEE. 